\title{
POLYNOMIAL ALGORITHMS FOR PROJECTING A POINT ONTO A REGION DEFINED BY A LINEAR CONSTRAINT AND BOX CONSTRAINTS IN $\mathbb{R}^{n}$
}

\author{
STEFAN M. STEFANOV
}

Received 29 September 2003 and in revised form 6 April 2004

We consider the problem of projecting a point onto a region defined by a linear equality or inequality constraint and two-sided bounds on the variables. Such problems are interesting because they arise in various practical problems and as subproblems of gradient-type methods for constrained optimization. Polynomial algorithms are proposed for solving these problems and their convergence is proved. Some examples and results of numerical experiments are presented.

\section{Introduction}

Consider the problem of projecting a point $\widehat{\mathbf{x}}=\left(\hat{x}_{1}, \ldots, \hat{x}_{n}\right) \in \mathbb{R}^{n}$ onto a set defined by a linear inequality constraint " $\leq$ ", linear equality constraint, or linear inequality constraint " $\geq$ " with positive coefficients and box constraints. This problem can be mathematically formulated as the following quadratic programming problem:

$$
\min \left\{c(\mathbf{x}) \equiv \sum_{j=1}^{n} c_{j}\left(x_{j}\right) \equiv \frac{1}{2} \sum_{j=1}^{n}\left(x_{j}-\hat{x}_{j}\right)^{2}\right\}
$$

subject to

$$
\mathbf{x} \in X,
$$

where the feasible region $X$ is defined by

$$
\begin{gathered}
\sum_{j=1}^{n} d_{j} x_{j} \leq \alpha, \quad d_{j}>0, j=1, \ldots, n, \\
a_{j} \leq x_{j} \leq b_{j}, \quad j=1, \ldots, n,
\end{gathered}
$$


in the first case, by

$$
\begin{gathered}
\sum_{j=1}^{n} d_{j} x_{j}=\alpha, \quad d_{j}>0, j=1, \ldots, n, \\
a_{j} \leq x_{j} \leq b_{j}, \quad j=1, \ldots, n,
\end{gathered}
$$

in the second case, or by

$$
\begin{gathered}
\sum_{j=1}^{n} d_{j} x_{j} \geq \alpha, \quad d_{j}>0, j=1, \ldots, n, \\
a_{j} \leq x_{j} \leq b_{j}, \quad j=1, \ldots, n,
\end{gathered}
$$

in the third case.

Denote this problem by $\left(P^{\leq}\right)$in the first case (problem (1.1)-(1.2) with $X$ defined by (1.3)-(1.4)), by $\left(P^{=}\right)$in the second case (problem (1.1)-(1.2) with $X$ defined by (1.5)(1.6)), and by $\left(P^{\geq}\right)$in the third case (problem (1.1)-(1.2) with $X$ defined by (1.7)-(1.8)).

Since $c(\mathbf{x})$ is a strictly convex function and $X$ is a convex closed set, then this is a convex programming problem and it always has a unique optimal solution when $X \neq \varnothing$.

Problems of the form (1.1)-(1.2) with $X$ defined by (1.3)-(1.4), (1.5)-(1.6), or (1.7)(1.8) arise in production planning and scheduling (see [2]), in allocation of resources (see $[2,7,8,15])$, in the theory of search (see $[4])$, in facility location (see $[10,11,12,13,14]$ ), and so forth. Problems $\left(P^{\leq}\right),\left(P^{=}\right)$, and $\left(P^{\geq}\right)$also arise as subproblems of some projection optimization methods of gradient (subgradient) type for constrained optimization when the feasible region is of the form (1.3)-(1.4), (1.5)-(1.6), or (1.7)-(1.8) (see, e.g., [6]). These projection problems are to be solved at each iteration of algorithm performance because current points generated by these methods must be projected on the feasible region at each iteration. That is why projection is the most onerous and time-consuming part of any projection gradient-type method for constrained optimization and we need efficient algorithms for solving these problems. This is the motivation to study the problems under consideration.

Problems like $\left(P^{\leq}\right),\left(P^{=}\right)$, and $\left(P^{\geq}\right)$are subject of intensive study. Related problems and methods for them are considered in $[1,2,3,4,5,6,7,8,9,10,11,12,13,14,15]$.

An algorithm for finding a projection onto a simple polytope is proposed, for example, in [9]. Projections in the implementation of stochastic quasigradient methods are studied in [10]. Projected Newton-type methods are suggested in [1,5].

This paper is devoted to the development of new efficient polynomial algorithms for finding a projection onto the set $X$ defined by (1.3)-(1.4), (1.5)-(1.6), or (1.7)-(1.8). The paper is organized as follows. In Section 2, characterization theorems (necessary and sufficient conditions or sufficient conditions) for the optimal solutions to the considered problems are proved. In Section 3, new algorithms of polynomial complexity are suggested and their convergence is proved. In Section 4, we consider some theoretical and numerical aspects of implementation of the algorithms and give some extensions of both 
characterization theorems and algorithms. In Section 5, we present results of some numerical experiments.

\section{Main results. Characterization theorems}

2.1. Problem $\left(P^{\leq}\right)$. First consider the following problem:

$\left(P^{\leq}\right)$

$$
\min \left\{c(\mathbf{x}) \equiv \sum_{j=1}^{n} c_{j}\left(x_{j}\right) \equiv \frac{1}{2} \sum_{j=1}^{n}\left(x_{j}-\hat{x}_{j}\right)^{2}\right\}
$$

subject to (1.3) and (1.4).

Suppose that the following assumptions are satisfied:

(1.a) $a_{j} \leq b_{j}$ for all $j=1, \ldots, n$. If $a_{k}=b_{k}$ for some $k, 1 \leq k \leq n$, then the value $x_{k}:=$ $a_{k}=b_{k}$ is determined in advance;

(1.b) $\sum_{j=1}^{n} d_{j} a_{j} \leq \alpha$; otherwise the constraints (1.3)-(1.4) are inconsistent and $X=\varnothing$ where $X$ is defined by (1.3)-(1.4).

In addition to this assumption we suppose that $\alpha \leq \sum_{j=1}^{n} d_{j} b_{j}$ in some cases which are specified below.

The Lagrangian for problem $\left(P^{\leq}\right)$is

$$
L(\mathbf{x}, \mathbf{u}, \mathbf{v}, \lambda)=\frac{1}{2} \sum_{j=1}^{n}\left(x_{j}-\hat{x}_{j}\right)^{2}+\lambda\left(\sum_{j=1}^{n} d_{j} x_{j}-\alpha\right)+\sum_{j=1}^{n} u_{j}\left(a_{j}-x_{j}\right)+\sum_{j=1}^{n} v_{j}\left(x_{j}-b_{j}\right),
$$

where $\lambda \in \mathbb{R}_{+}^{1}, \mathbf{u}, \mathbf{v} \in \mathbb{R}_{+}^{n}$, and $\mathbb{R}_{+}^{n}$ consists of all vectors with $n$ real nonnegative components.

The Karush-Kuhn-Tucker (KKT) necessary and sufficient optimality conditions for the minimum $\mathbf{x}^{*}=\left(x_{1}^{*}, \ldots, x_{n}^{*}\right)$ are

$$
\begin{gathered}
x_{j}^{*}-\hat{x}_{j}+\lambda d_{j}-u_{j}+v_{j}=0, \quad j=1, \ldots, n, \\
u_{j}\left(a_{j}-x_{j}^{*}\right)=0, \quad j=1, \ldots, n, \\
v_{j}\left(x_{j}^{*}-b_{j}\right)=0, \quad j=1, \ldots, n, \\
\lambda\left(\sum_{j=1}^{n} d_{j} x_{j}^{*}-\alpha\right)=0, \quad \lambda \in \mathbb{R}_{+}^{1}, \\
\sum_{j=1}^{n} d_{j} x_{j}^{*} \leq \alpha, \\
a_{j} \leq x_{j}^{*} \leq b_{j}, \quad j=1, \ldots, n, \\
u_{j} \in \mathbb{R}_{+}^{1}, \quad v_{j} \in \mathbb{R}_{+}^{1}, \quad j=1, \ldots, n .
\end{gathered}
$$


Here, $\lambda, u_{j}, v_{j}, j=1, \ldots, n$, are the Lagrange multipliers associated with the constraints (1.3), $a_{j} \leq x_{j}, x_{j} \leq b_{j}, j=1, \ldots, n$, respectively. If $a_{j}=-\infty$ or $b_{j}=+\infty$ for some $j$, we do not consider the corresponding condition (2.4), (2.5) and Lagrange multiplier $u_{j}\left[v_{j}\right]$.

Since $\lambda \geq 0, u_{j} \geq 0, v_{j} \geq 0, j=1, \ldots, n$, and since the complementary conditions (2.4), (2.5), (2.6) must be satisfied, in order to find $x_{j}^{*}, j=1, \ldots, n$, from system (2.3)-(2.9), we have to consider all possible cases for $\lambda, u_{j}, v_{j}$ : all $\lambda, u_{j}, v_{j}$ equal to 0 ; all $\lambda, u_{j}, v_{j}$ different from 0 ; some of them equal to 0 and some of them different from 0 . The number of these cases is $2^{2 n+1}$, where $2 n+1$ is the number of $\lambda, u_{j}, v_{j}, j=1, \ldots, n$. Obviously this is an enormous number of cases, especially for large-scale problems. For example, when $n=1500$, we have $2^{3001} \approx 10^{900}$ cases. Moreover, in each case we have to solve a large-scale system of (nonlinear) equations in $x_{j}^{*}, \lambda, u_{j}, v_{j}, j=1, \ldots, n$. Therefore direct application of the KKT theorem, using explicit enumeration of all possible cases, for solving largescale problems of the considered form would not give a result and we need results and efficient methods to cope with these problems.

The following theorem gives a characterization of the optimal solution to problem $\left(P^{\leq}\right)$. Its proof, of course, is based on the KKT theorem. As we will see in Section 5, by using Theorem 2.1, we can solve problem $\left(P^{\leq}\right)$with $n=10000$ variables in 0.00055 seconds on a personal computer.

Theorem 2.1 (characterization of the optimal solution to problem $\left(P^{\leq}\right)$). A feasible solution $\mathbf{x}^{*}=\left(x_{1}^{*}, \ldots, x_{n}^{*}\right) \in X(1.3)$-(1.4) is the optimal solution to problem $\left(P^{\leq}\right)$if and only if there exists some $\lambda \in \mathbb{R}_{+}^{1}$ such that

$$
\begin{aligned}
& x_{j}^{*}=a_{j}, \quad j \in J_{a}^{\lambda} \stackrel{\text { def }}{=}\left\{j: \lambda \geq \frac{\hat{x}_{j}-a_{j}}{d_{j}}\right\}, \\
& x_{j}^{*}=b_{j}, \quad j \in J_{b}^{\lambda} \stackrel{\text { def }}{=}\left\{j: \lambda \leq \frac{\hat{x}_{j}-b_{j}}{d_{j}}\right\}, \\
& x_{j}^{*}=\hat{x}_{j}-\lambda d_{j}, \quad j \in J^{\lambda} \stackrel{\text { def }}{=}\left\{j: \frac{\hat{x}_{j}-b_{j}}{d_{j}}<\lambda<\frac{\hat{x}_{j}-a_{j}}{d_{j}}\right\} .
\end{aligned}
$$

Proof. (i) Let $\mathbf{x}^{*}=\left(x_{1}^{*}, \ldots, x_{n}^{*}\right)$ be the optimal solution to $\left(P^{\leq}\right)$. Then there exist constants $\lambda, u_{j}, v_{j}, j=1, \ldots, n$, such that the KKT conditions (2.3)-(2.9) are satisfied. Consider both possible cases for $\lambda$.

(1) Let $\lambda>0$. Then system (2.3)-(2.9) becomes (2.3), (2.4), (2.5), (2.8), (2.9), and

$$
\sum_{j=1}^{n} d_{j} x_{j}^{*}=\alpha,
$$

that is, the inequality constraint (1.3) is satisfied with an equality for $x_{j}^{*}, j=$ $1, \ldots, n$, in this case.

(a) If $x_{j}^{*}=a_{j}$, then $u_{j} \geq 0$ and $v_{j}=0$ according to (2.5). Therefore (2.3) implies $x_{j}^{*}-\hat{x}_{j}=u_{j}-\lambda d_{j} \geq-\lambda d_{j}$. Since $d_{j}>0$, then

$$
\lambda \geq \frac{\hat{x}_{j}-x_{j}^{*}}{d_{j}} \equiv \frac{\hat{x}_{j}-a_{j}}{d_{j}} .
$$


(b) If $x_{j}^{*}=b_{j}$, then $u_{j}=0$ according to (2.4) and $v_{j} \geq 0$. Therefore (2.3) implies $x_{j}^{*}-\hat{x}_{j}=-v_{j}-\lambda d_{j} \leq-\lambda d_{j}$. Hence

$$
\lambda \leq \frac{\hat{x}_{j}-x_{j}^{*}}{d_{j}} \equiv \frac{\hat{x}_{j}-b_{j}}{d_{j}} .
$$

(c) If $a_{j}<x_{j}^{*}<b_{j}$, then $u_{j}=v_{j}=0$ according to (2.4) and (2.5). Therefore (2.3) implies $x_{j}^{*}=\hat{x}_{j}-\lambda d_{j}$. Since $d_{j}>0, j=1, \ldots, n, \lambda>0$ by the assumptions, then $\hat{x}_{j}>x_{j}^{*}$. It follows from $b_{j}>x_{j}^{*}, x_{j}^{*}>a_{j}$ that

$$
\hat{x}_{j}-b_{j}<\hat{x}_{j}-x_{j}^{*}, \quad \hat{x}_{j}-x_{j}^{*}<\hat{x}_{j}-a_{j} .
$$

Using $d_{j}>0$, we obtain $\lambda=\left(\hat{x}_{j}-x_{j}^{*}\right) / d_{j}<\left(\hat{x}_{j}-a_{j}\right) / d_{j}, \lambda=\left(\hat{x}_{j}-x_{j}^{*}\right) / d_{j}>$ $\left(\hat{x}_{j}-b_{j}\right) / d_{j}$, that is,

$$
\frac{\hat{x}_{j}-b_{j}}{d_{j}}<\lambda<\frac{\hat{x}_{j}-a_{j}}{d_{j}} .
$$

(2) Let $\lambda=0$. Then system (2.3)-(2.9) becomes

$$
x_{j}^{*}-\hat{x}_{j}-u_{j}+v_{j}=0, \quad j=1, \ldots, n,
$$

and (2.4), (2.5), (2.7), (2.8), (2.9).

(a) If $x_{j}^{*}=a_{j}$, then $u_{j} \geq 0, v_{j}=0$. Therefore $a_{j}-\hat{x}_{j} \equiv x_{j}^{*}-\hat{x}_{j}=u_{j} \geq 0$. Multiplying both sides of this inequality by $-\left(1 / d_{j}\right)(<0$ by the assumption), we obtain

$$
\frac{\hat{x}_{j}-a_{j}}{d_{j}} \leq 0 \equiv \lambda .
$$

(b) If $x_{j}^{*}=b_{j}$, then $u_{j}=0, v_{j} \geq 0$. Therefore $b_{j}-\hat{x}_{j} \equiv x_{j}^{*}-\hat{x}_{j}=-v_{j} \leq 0$. Multiplying this inequality by $-\left(1 / d_{j}\right)<0$, we get

$$
\frac{\hat{x}_{j}-b_{j}}{d_{j}} \geq 0 \equiv \lambda \text {. }
$$

(c) If $a_{j}<x_{j}^{*}<b_{j}$, then $u_{j}=v_{j}=0$. Therefore $x_{j}^{*}-\hat{x}_{j}=0$, that is, $x_{j}^{*}=\hat{x}_{j}$. Since $b_{j}>x_{j}^{*}, x_{j}^{*}>a_{j}, j=1, \ldots, n$, by the assumption, then

$$
\hat{x}_{j}-b_{j}<\hat{x}_{j}-x_{j}^{*}=0, \quad 0=\hat{x}_{j}-x_{j}^{*}<\hat{x}_{j}-a_{j} .
$$

Multiplying both inequalities by $1 / d_{j}>0$, we obtain

$$
\frac{\hat{x}_{j}-b_{j}}{d_{j}}<0 \equiv \lambda, \quad \lambda \equiv 0<\frac{\hat{x}_{j}-a_{j}}{d_{j}},
$$

that is, in case (c) we have

$$
\frac{\hat{x}_{j}-b_{j}}{d_{j}}<\lambda<\frac{\hat{x}_{j}-a_{j}}{d_{j}} .
$$


In order to describe cases (a), (b), (c) for both (1) and (2), it is convenient to introduce the index sets $J_{a}^{\lambda}, J_{b}^{\lambda}, J^{\lambda}$ defined by (2.10), (2.11), and (2.12), respectively. Obviously $J_{a}^{\lambda} \cup$ $J_{b}^{\lambda} \cup J^{\lambda}=\{1, \ldots, n\}$. The "necessity" part is proved.

(ii) Conversely, let $\mathbf{x}^{*} \in X$ and let components of $\mathbf{x}^{*}$ satisfy (2.10), (2.11), and (2.12), where $\lambda \in \mathbb{R}_{+}^{1}$.

(1) If $\lambda>0$, then $x_{j}^{*}-\hat{x}_{j}<0, j \in J^{\lambda}$, according to (2.12) and $d_{j}>0$. Set

$\lambda=\frac{\hat{x}_{j}-x_{j}^{*}}{d_{j}}(>0)$ obtained from $\sum_{j \in J_{a}^{\lambda}} d_{j} a_{j}+\sum_{j \in J_{b}^{\lambda}} d_{j} b_{j}+\sum_{j \in J^{\lambda}} d_{j}\left(\hat{x}_{j}-\lambda d_{j}\right)=\alpha ;$

$u_{j}=v_{j}=0 \quad$ for $j \in J^{\lambda}$;

$u_{j}=a_{j}-\hat{x}_{j}+\lambda d_{j}\left(\geq 0\right.$ according to the definition of $\left.J_{a}^{\lambda}\right), \quad v_{j}=0 \quad$ for $j \in J_{a}^{\lambda}$;

$u_{j}=0, \quad v_{j}=\hat{x}_{j}-b_{j}-\lambda d_{j}\left(\geq 0\right.$ according to the definition of $\left.J_{b}^{\lambda}\right) \quad$ for $j \in J_{b}^{\lambda}$.

By using these expressions, it is easy to check that conditions (2.3), (2.4), (2.5), (2.6), (2.9) are satisfied; conditions (2.7) and (2.8) are also satisfied according to the assumption $\mathbf{x}^{*} \in X$.

(2) If $\lambda=0$, then $x_{j}^{*}=\hat{x}_{j}, j \in J^{\lambda}$, according to (2.12), and

$$
J^{\lambda=0}=\left\{j: \frac{\hat{x}_{j}-b_{j}}{d_{j}}<0<\frac{\hat{x}_{j}-a_{j}}{d_{j}}\right\} .
$$

Since $d_{j}>0, \hat{x}_{j}-b_{j}<0, \hat{x}_{j}-a_{j}>0, j \in J^{0}$. Therefore $x_{j}^{*}=\hat{x}_{j} \in\left(a_{j}, b_{j}\right)$. Set

$$
\begin{gathered}
\lambda=\frac{\hat{x}_{j}-x_{j}^{*}}{d_{j}}(=0), \quad u_{j}=v_{j}=0 \quad \text { for } j \in J^{\lambda=0}, \\
u_{j}=a_{j}-\hat{x}_{j}+\lambda d_{j}=a_{j}-\hat{x}_{j}(\geq 0), \quad v_{j}=0 \quad \text { for } j \in J_{a}^{\lambda=0}, \\
u_{j}=0, \quad v_{j}=\hat{x}_{j}-b_{j}-\lambda d_{j}=\hat{x}_{j}-b_{j}(\geq 0)
\end{gathered}
$$

Obviously conditions (2.3), (2.4), (2.5), (2.9) are satisfied; conditions (2.7), (2.8) are also satisfied according to the assumption $\mathbf{x}^{*} \in X$, and condition (2.6) is obviously satisfied for $\lambda=0$.

In both cases (1) and (2) of part (ii), $x_{j}^{*}, \lambda, u_{j}, v_{j}, j=1, \ldots, n$, satisfy KKT conditions (2.3)-(2.9) which are necessary and sufficient conditions for a feasible solution to be an optimal solution to a convex minimization problem. Therefore $\mathbf{x}^{*}$ is the (unique) optimal solution to problem $\left(P^{\leq}\right)$.

In view of the discussion above, the importance of Theorem 2.1 consists in the fact that it describes components of the optimal solution to $\left(P^{\leq}\right)$only through the Lagrange multiplier $\lambda$ associated with the inequality constraint (1.3). 
Since we do not know the optimal value of $\lambda$ from Theorem 2.1, we define an iterative process with respect to the Lagrange multiplier $\lambda$ and we prove convergence of this process in Section 3.

It follows from $d_{j}>0$ and $a_{j} \leq b_{j}, j=1, \ldots, n$, that

$$
u b_{j} \stackrel{\text { def }}{=} \frac{\hat{x}_{j}-b_{j}}{d_{j}} \leq \frac{\hat{x}_{j}-a_{j}}{d_{j}} \stackrel{\text { def }}{=} l a_{j}, \quad j=1, \ldots, n,
$$

for the expressions by means of which we define the sets $J_{a}^{\lambda}, J_{b}^{\lambda}, J^{\lambda}$.

The problem how to ensure a feasible solution to problem $\left(P^{\leq}\right)$, which is an assumption of Theorem 2.1, is discussed after the statement of the corresponding algorithm.

2.2. Problem $\left(P^{=}\right)$. Consider problem $\left(P^{=}\right)$of finding a projection of $\hat{\mathbf{x}}$ onto a set $X$ of the form (1.5)-(1.6):

$\left(P^{=}\right)$

$$
\min \left\{c(\mathbf{x}) \equiv \sum_{j=1}^{n} c_{j}\left(x_{j}\right) \equiv \frac{1}{2} \sum_{j=1}^{n}\left(x_{j}-\hat{x}_{j}\right)^{2}\right\}
$$

subject to (1.5) and (1.6).

We have the following assumptions:

(2.a) $a_{j} \leq b_{j}$ for all $j=1, \ldots, n$;

(2.b) $\sum_{j=1}^{n} d_{j} a_{j} \leq \alpha \leq \sum_{j=1}^{n} d_{j} b_{j}$; otherwise the constraints (1.5)-(1.6) are inconsistent and the feasible region (1.5)-(1.6) is empty.

The KKT conditions for problem $\left(P^{=}\right)$are

$$
\begin{gathered}
x_{j}^{*}-\hat{x}_{j}+\lambda d_{j}-u_{j}+v_{j}=0, \quad j=1, \ldots, n, \lambda \in \mathbb{R}^{1}, \\
u_{j}\left(a_{j}-x_{j}^{*}\right)=0, \quad j=1, \ldots, n, \\
v_{j}\left(x_{j}^{*}-b_{j}\right)=0, \quad j=1, \ldots, n, \\
\sum_{j=1}^{n} d_{j} x_{j}^{*}=\alpha, \\
a_{j} \leq x_{j}^{*} \leq b_{j}, \quad j=1, \ldots, n, \\
u_{j} \in \mathbb{R}_{+}^{1}, \quad v_{j} \in \mathbb{R}_{+}^{1}, \quad j=1, \ldots, n .
\end{gathered}
$$

In this case the following theorem, which is analogous to Theorem 2.1, holds true.

Theorem 2.2 (characterization of the optimal solution to problem $\left(P^{=}\right)$). A feasible solution $\mathbf{x}^{*}=\left(x_{1}^{*}, \ldots, x_{n}^{*}\right) \in X(1.5)-(1.6)$ is the optimal solution to problem $\left(P^{=}\right)$if and only 
if there exists some $\lambda \in \mathbb{R}^{1}$ such that

$$
\begin{aligned}
& x_{j}^{*}=a_{j}, \quad j \in J_{a}^{\lambda} \stackrel{\text { def }}{=}\left\{j: \lambda \geq \frac{\hat{x}_{j}-a_{j}}{d_{j}}\right\}, \\
& x_{j}^{*}=b_{j}, \quad j \in J_{b}^{\lambda} \stackrel{\text { def }}{=}\left\{j: \lambda \leq \frac{\hat{x}_{j}-b_{j}}{d_{j}}\right\}, \\
& x_{j}^{*}=\hat{x}_{j}-\lambda d_{j}, \quad j \in J^{\lambda} \stackrel{\text { def }}{=}\left\{j: \frac{\hat{x}_{j}-b_{j}}{d_{j}}<\lambda<\frac{\hat{x}_{j}-a_{j}}{d_{j}}\right\} .
\end{aligned}
$$

The proof of Theorem 2.2 is omitted because it is similar to that of Theorem 2.1.

2.3. Problem $\left(P^{\geq}\right)$. Consider problem $\left(P^{\geq}\right)$of finding a projection of $\hat{\mathbf{x}}$ onto a set $X$ of the form (1.7)-(1.8):

$\left(P^{\geq}\right)$

$$
\min \left\{c(\mathbf{x}) \equiv \sum_{j=1}^{n} c_{j}\left(x_{j}\right) \equiv \frac{1}{2} \sum_{j=1}^{n}\left(x_{j}-\hat{x}_{j}\right)^{2}\right\}
$$

subject to (1.7) and (1.8).

We have the following assumptions:

(3.a) $a_{j} \leq b_{j}$ for all $j=1, \ldots, n$;

(3.b) $\alpha \leq \sum_{j=1}^{n} d_{j} b_{j}$; otherwise constraints (1.7)-(1.8) are inconsistent and $X=\varnothing$, where $X$ is defined by (1.7)-(1.8).

Rewrite $\left(P^{\geq}\right)$in the form (2.33), (2.34), (1.8), where

$$
-\sum_{j=1}^{n} d_{j} x_{j} \leq-\alpha, \quad d_{j}>0, j=1, \ldots, n .
$$

Since the linear function $d(\mathbf{x}):=-\sum_{j=1}^{n} d_{j} x_{j}+\alpha$ is both convex and concave, $\left(P^{\geq}\right)$is a convex optimization problem.

Let $\lambda, \lambda^{\geq}$be the Lagrange multipliers associated with (1.5) (problem $\left(P^{=}\right)$) and with (2.34) (problem $\left(P^{\geq}\right)$), and let $x_{j}^{*}, x_{j}^{\geq}, j=1, \ldots, n$, be components of the optimal solutions to $\left(P^{=}\right),\left(P^{\geq}\right)$, respectively. For the sake of simplicity, we use $u_{j}, v_{j}, j=1, \ldots, n$, instead of $u_{j}^{\geq}, v_{j}^{\geq}, j=1, \ldots, n$, for the Lagrange multipliers associated with $a_{j} \leq x_{j}, x_{j} \leq b_{j}$, $j=1, \ldots, n$, from $(1.8)$, respectively.

The Lagrangian for problem $\left(P^{\geq}\right)$is

$$
\begin{aligned}
L\left(\mathbf{x}, \mathbf{u}, \mathbf{v}, \lambda^{\geq}\right)= & \frac{1}{2} \sum_{j=1}^{n}\left(x_{j}-\hat{x}_{j}\right)^{2}+\lambda^{\geq}\left(-\sum_{j=1}^{n} d_{j} x_{j}+\alpha\right) \\
& +\sum_{j=1}^{n} u_{j}\left(a_{j}-x_{j}\right)+\sum_{j=1}^{n} v_{j}\left(x_{j}-b_{j}\right)
\end{aligned}
$$


and the KKT conditions for $\left(P^{\geq}\right)$are

$$
\begin{gathered}
x_{j}^{\geq}-\hat{x}_{j}-\lambda^{\geq} d_{j}-u_{j}+v_{j}=0, \quad j=1, \ldots, n, \\
u_{j}\left(a_{j}-x_{j}^{\geq}\right)=0, \quad j=1, \ldots, n, \\
v_{j}\left(x_{j}^{\geq}-b_{j}\right)=0, \quad j=1, \ldots, n, \\
\lambda^{\geq}\left(\alpha-\sum_{j=1}^{n} d_{j} x_{j}^{\geq}\right)=0, \quad \lambda^{\geq} \in \mathbb{R}_{+}^{1}, \\
-\sum_{j=1}^{n} d_{j} x_{j}^{\geq} \leq-\alpha, \\
a_{j} \leq x_{j}^{\geq} \leq b_{j}, \quad j=1, \ldots, n, \\
u_{j} \in \mathbb{R}_{+}^{1}, \quad v_{j} \in \mathbb{R}_{+}^{1}, \quad j=1, \ldots, n .
\end{gathered}
$$

We can replace (2.36) and (2.39) by

$$
\begin{aligned}
& x_{j}^{\geq}-\hat{x}_{j}+\lambda^{\geq} d_{j}-u_{j}+v_{j}=0, \quad j=1, \ldots, n, \\
& \lambda^{\geq}\left(\sum_{j=1}^{n} d_{j} x_{j}^{\geq}-\alpha\right)=0, \quad \lambda^{\geq} \in \mathbb{R}_{-}^{1}, d_{j}>0,
\end{aligned}
$$

respectively, where we have redenoted $\lambda^{\geq}:=-\lambda^{\geq} \in \mathbb{R}_{-}^{1}$.

Conditions (2.43) with $\lambda$ instead of $\lambda^{\geq},(2.37),(2.38),(2.41),(2.42)$ are among the KKT conditions for problem $\left(P^{=}\right)$.

Theorem 2.3 (sufficient condition for optimal solution). (i) If $\lambda=\left(\hat{x}_{j}-x_{j}^{*}\right) / d_{j} \leq 0$, then $x_{j}^{*}, j=1, \ldots, n$, solve problem $\left(P^{\geq}\right)$as well.

(ii) If $\lambda=\left(\hat{x}_{j}-x_{j}^{*}\right) / d_{j}>0$, then $x_{j}^{>}, j=1, \ldots, n$, defined as

$$
\begin{gathered}
x_{j}^{\geq}=b_{j}, \quad j \in J_{b}^{\lambda} ; \\
x_{j}^{\geq}=\min \left\{b_{j}, \hat{x}_{j}\right\}, \quad j \in J^{\lambda} ; \\
x_{j}^{\geq}=\min \left\{b_{j}, \hat{x}_{j}\right\} \quad \forall j \in J_{a}^{\lambda} \text { such that } a_{j}<\hat{x}_{j} ; \\
x_{j}^{\geq}=a_{j} \quad \forall j \in J_{a}^{\lambda} \text { such that } a_{j} \geq \hat{x}_{j}
\end{gathered}
$$

solve problem $\left(P^{\geq}\right)$.

Proof. (i) Let $\lambda=\left(\hat{x}_{j}-x_{j}^{*}\right) / d_{j} \leq 0$ (i.e., $\hat{x}_{j} \leq x_{j}^{*}, j \in J^{\lambda}$, because $\left.d_{j}>0\right)$. Since $x_{j}^{*}, j=$ $1, \ldots, n$, satisfy KKT conditions for problem $\left(P^{=}\right)$as components of the optimal solution to $\left(P^{=}\right)$, then (2.43), (2.37), (2.38), (2.40) with equality (and therefore (2.44)), (2.41), (2.42) are satisfied as well (with $\lambda$ instead of $\lambda^{\geq}$). Since they are the KKT necessary and sufficient conditions for $\left(P^{\geq}\right)$, then $x_{j}^{*}, j=1, \ldots, n$, solve $\left(P^{\geq}\right)$.

(ii) Let $\lambda=\left(\hat{x}_{j}-x_{j}^{*}\right) / d_{j}>0$ (i.e., $\left.\hat{x}_{j}>x_{j}^{*}, j \in J^{\lambda}\right)$. Since $\mathbf{x}^{*}=\left(x_{j}^{*}\right)_{j=1}^{n}$ is the optimal solution to $\left(P^{=}\right)$by the assumption, then KKT conditions for $\left(P^{=}\right)$are satisfied. 
If $\mathbf{x}^{\geq}:=\left(x_{j}^{\geq}\right)_{j=1}^{n}$ is the optimal solution to $\left(P^{\geq}\right)$, then $\mathbf{x}^{\geq}$satisfies (2.43), (2.37), (2.38), (2.44), (2.40), (2.41), (2.42). Since $\lambda>0$, then $\lambda$ cannot play the role of $\lambda^{\geq}$in (2.43) and (2.44) because $\lambda^{\geq}$must be a nonpositive real number in (2.43) and (2.44). Therefore $x_{j}^{*}$, which satisfy KKT conditions for problem $\left(P^{=}\right)$, cannot play the roles of $x_{j}^{2}, j=1, \ldots, n$, in (2.43), (2.37), (2.38), (2.44), (2.40), (2.41), (2.42). Hence, in the general case the equality $\sum_{j=1}^{n} d_{j} x_{j}=\alpha$ is not satisfied for $x_{j}=x_{j}^{2}$. Therefore, in order that (2.44) be satisfied, $\lambda \geq$ must be equal to 0 . This conclusion helps us to prove the theorem.

Let $\mathbf{x}^{\geq}:=\left(x_{j}^{\geq}\right)_{j=1}^{n}$ be defined as in part (ii) of the statement of Theorem 2.3.

Set $\lambda^{\geq}=0$;

(1) $u_{j}=0, v_{j}=\hat{x}_{j}-b_{j}\left(\geq 0\right.$ according to the definition of $\left.J_{b}^{\lambda}(2.31), \lambda>0, d_{j}>0\right)$ for $j \in J_{b}^{\lambda}$

(2) $u_{j}=v_{j}=0$ for $j \in J_{a}^{\lambda}$ such that $a_{j}<\hat{x}_{j}$ and for $j \in J^{\lambda}$ such that $\hat{x}_{j}<b_{j}$;

(3) $u_{j}=0, v_{j}=\hat{x}_{j}-b_{j}(\geq 0)$ for $j \in J^{\lambda}$ such that $\hat{x}_{j} \geq b_{j}$;

(4) $u_{j}=a_{j}-\hat{x}_{j}(\geq 0), v_{j}=0$ for $j \in J_{a}^{\lambda}$ such that $a_{j} \geq \hat{x}_{j}$.

In case (2) we have $a_{j}<\hat{x}_{j}$, therefore $a_{j}<\hat{x}_{j}=x_{j}^{\geq}$according to the definition of $x_{j}^{\geq}$in this case. In case (3), since $b_{j} \leq \hat{x}_{j}$, that is, $b_{j}-\hat{x}_{j} \leq 0$, then $v_{j}:=\hat{x}_{j}-b_{j} \geq 0$. Consequently, conditions (2.41) and (2.42) are satisfied for all $j$ according to (1), (2), (3), and (4).

As we have proved, (2.44) is satisfied with $\lambda^{\geq}=0$. Since the equality constraint (1.5) $\sum_{j=1}^{n} d_{j} x_{j}^{*}=\alpha$ is satisfied for the optimal solution $\mathbf{x}^{*}$ to $\left(P^{=}\right)$, since the components of $\mathbf{x}^{\geq}$ defined in the statement of Theorem 2.3(ii) are such that some of them are the same as the corresponding components of $\mathbf{x}^{*}$, since some of the components of $\mathbf{x}^{\geq}$, namely those for $j \in J_{a}^{\lambda}$ with $a_{j}<\hat{x}_{j}$, are greater than the corresponding components $x_{j}^{*}=a_{j}, j \in J_{a}^{\lambda}$, of $\mathbf{x}^{*}$, and since $d_{j}>0, j=1, \ldots, n$, then obviously the inequality constraint (2.40) holds for $\mathbf{x}^{\geq}$. It is easy to check that other conditions (2.43), (2.37), (2.38) are also satisfied. Thus, $x_{j}^{\geq}, j=1, \ldots, n$, defined above satisfy the KKT conditions for $\left(P^{\geq}\right)$. Therefore $\mathbf{x}^{\geq}$is the optimal solution to problem $\left(P^{\geq}\right)$.

According to Theorem 2.3, the optimal solution to problem $\left(P^{\geq}\right)$is obtained by using the optimal solution and optimal value of the Lagrange multiplier $\lambda$ for problem $\left(P^{=}\right)$. That is why we suppose that $\sum_{j=1}^{n} d_{j} a_{j} \leq \alpha$ in addition to assumption (3.b) (see Step (1) of Algorithm 3 below) as we assumed this in assumption (2.b) for problem $\left(P^{=}\right)$.

\section{The algorithms}

3.1. Analysis of the optimal solution to problem $\left(P^{\leq}\right)$. Before the formal statement of the algorithm for problem $\left(P^{\leq}\right)$, we discuss some properties of the optimal solution to this problem, which turn out to be useful.

Using (2.10), (2.11), and (2.12), condition (2.6) can be written as follows:

$$
\lambda\left(\sum_{j \in J_{a}^{\lambda}} d_{j} a_{j}+\sum_{j \in J_{b}^{\lambda}} d_{j} b_{j}+\sum_{j \in J^{\lambda}} d_{j}\left(\hat{x}_{j}-\lambda d_{j}\right)-\alpha\right)=0, \quad \lambda \geq 0
$$

Since the optimal solution $\mathbf{x}^{*}$ to problem $\left(P^{\leq}\right)$obviously depends on $\lambda$, we consider 
components of $\mathbf{x}^{*}$ as functions of $\lambda$ for different $\lambda \in \mathbb{R}_{+}^{1}$ :

$$
x_{j}^{*}=x_{j}(\lambda)= \begin{cases}a_{j}, & j \in J_{a}^{\lambda}, \\ b_{j}, & j \in J_{b}^{\lambda}, \\ \hat{x}_{j}-\lambda d_{j}, & j \in J^{\lambda} .\end{cases}
$$

Functions $x_{j}(\lambda), j=1, \ldots, n$, are piecewise linear, monotone nonincreasing, piecewise differentiable functions of $\lambda$ with two breakpoints at $\lambda=\left(\hat{x}_{j}-a_{j}\right) / d_{j}$ and $\lambda=\left(\hat{x}_{j}-b_{j}\right) / d_{j}$.

Let

$$
\delta(\lambda) \stackrel{\text { def }}{=} \sum_{j \in J_{a}^{\lambda}} d_{j} a_{j}+\sum_{j \in J_{b}^{\lambda}} d_{j} b_{j}+\sum_{j \in J^{\lambda}} d_{j} \hat{x}_{j}-\lambda \sum_{j \in J^{\lambda}} d_{j}^{2}-\alpha .
$$

If we differentiate (3.3) with respect to $\lambda$, we get

$$
\delta^{\prime}(\lambda) \equiv-\sum_{j \in J^{\lambda}} d_{j}^{2}<0,
$$

when $J^{\lambda} \neq \varnothing$, and $\delta^{\prime}(\lambda)=0$ when $J^{\lambda}=\varnothing$. Hence $\delta(\lambda)$ is a monotone nonincreasing function of $\lambda, \lambda \in \mathbb{R}_{+}^{1}$, and $\max _{\lambda \geq 0} \delta(\lambda)$ is attained at the minimum admissible value of $\lambda$, that is, at $\lambda=0$.

Case 1. If $\delta(0)>0$, in order that (3.1) and (2.7) be satisfied, there exists some $\lambda^{*}>0$ such that $\delta\left(\lambda^{*}\right)=0$, that is,

$$
\sum_{j=1}^{n} d_{j} x_{j}^{*}=\alpha
$$

which means that the inequality constraint (1.3) is satisfied with an equality for $\lambda^{*}$ in this case.

Case 2. If $\delta(0)<0$, then $\delta(\lambda)<0$ for all $\lambda \geq 0$, and the maximum of $\delta(\lambda)$ with $\lambda \geq 0$ is $\delta(0)=\max _{\lambda \geq 0} \delta(\lambda)$ and it is attained at $\lambda=0$ in this case. In order that (3.1) be satisfied, $\lambda$ must be equal to 0 . Therefore $x_{j}^{*}=\hat{x}_{j}, j \in J^{\lambda=0}$, according to (2.12).

Case 3. In the special case when $\delta(0)=0$, the maximum $\delta(0)=\max _{\lambda \geq 0} \delta(\lambda)$ of $\delta(\lambda)$ is also attained at the minimum admissible value of $\lambda$, that is, for $\lambda=0$, because $\delta(\lambda)$ is a monotone nonincreasing function in accordance with the above consideration.

As we have seen, for the optimal value of $\lambda$, we have $\lambda \geq 0$ in all possible cases, as the KKT condition (2.6) requires. We have shown that in Case 1 we need an algorithm for finding $\lambda^{*}$ which satisfies the KKT conditions (2.3)-(2.9) but such that $\lambda^{*}$ satisfies (2.7) with an equality. In order that this be fulfilled, the set (1.5)-(1.6) (i.e., feasible region of problem $\left.\left(P^{=}\right)\right)$must be nonempty. That is why we have required $\alpha \leq \sum_{j=1}^{n} d_{j} b_{j}$ in some cases in addition to the assumption $\sum_{j=1}^{n} d_{j} a_{j} \leq \alpha$ (see assumption (1.b)). We have also used this in the proof of Theorem 2.1, part (ii), when $\lambda>0$. 
Using the equation $\delta(\lambda)=0$, where $\delta(\lambda)$ is defined by (3.3), we are able to obtain a closed-form expression for $\lambda$

$$
\lambda=\left(\sum_{j \in J^{\lambda}} d_{j}^{2}\right)^{-1}\left(\sum_{j \in J_{a}^{\lambda}} d_{j} a_{j}+\sum_{j \in J_{b}^{\lambda}} d_{j} b_{j}+\sum_{j \in J^{\lambda}} d_{j} \hat{x}_{j}-\alpha\right),
$$

because $\delta^{\prime}(\lambda)<0$ according to (3.4) when $J^{\lambda} \neq \varnothing$ (it is important that $\delta^{\prime}(\lambda) \neq 0$ ). This expression of $\lambda$ is used in the algorithm suggested for problem $\left(P^{\leq}\right)$. It turns out that for our purposes, without loss of generality, we can assume that $\delta^{\prime}(\lambda) \neq 0$, that is, $\delta(\lambda)$ depends on $\lambda$, which means that $J^{\lambda} \neq \varnothing$.

At iteration $k$ of the implementation of the algorithms, denote by $\lambda^{(k)}$ the value of the Lagrange multiplier associated with constraint (1.3) (resp., (1.5), (1.7)), by $\alpha^{(k)}$ the right-hand side of (1.3) (resp., (1.5), (1.7)), and by $J^{(k)}, J_{a}^{\lambda(k)}, J_{b}^{\lambda(k)}, J^{\lambda(k)}$ the current sets $J=\{1, \ldots, n\}, J_{a}^{\lambda}, J_{b}^{\lambda}, J^{\lambda}$, respectively.

3.2. Algorithm 1. The following algorithm for solving problem $\left(P^{\leq}\right)$is based on Theorem 2.1 .

Algorithm 1 (for problem $\left(P^{\leq}\right)$).

(0) (Initialization). $J:=\{1, \ldots, n\}, k:=0, \alpha^{(0)}:=\alpha, n^{(0)}:=n, J^{(0)}:=J, J_{a}^{\lambda}:=\varnothing, J_{b}^{\lambda}:=\varnothing$, initialize $\hat{x}_{j}, j \in J$. If $\sum_{j \in J} d_{j} a_{j} \leq \alpha$, go to (1) else go to (9).

(1) Construct the sets $J_{a}^{0}, J_{b}^{0}, J^{0}$ (for $\lambda=0$ ). Calculate

$$
\delta(0):=\sum_{j \in J_{a}^{0}} d_{j} a_{j}+\sum_{j \in J_{b}^{0}} d_{j} b_{j}+\sum_{j \in J^{0}} d_{j} \hat{x}_{j}-\alpha .
$$

If $\delta(0) \leq 0$, then $\lambda:=0$, go to $(8)$

else if $\delta(0)>0$, then

if $\alpha \leq \sum_{j \in J} d_{j} b_{j}$, go to (2)

else if $\alpha>\sum_{j \in J} d_{j} b_{j}$, go to (9) (there does not exist $\lambda^{*}>0$ such that $\delta\left(\lambda^{*}\right)=0$ ).

(2) $J^{\lambda(k)}:=J^{(k)}$. Calculate $\lambda^{(k)}$ by using the explicit expression of $\lambda$ (3.6). Go to (3).

(3) Construct the sets $J_{a}^{\lambda(k)}, J_{b}^{\lambda(k)}, J^{\lambda(k)}$ through (2.10), (2.11), (2.12) (with $j \in J^{(k)}$ instead of $j \in J)$ and find their cardinalities $\left|J_{a}^{\lambda(k)}\right|,\left|J_{b}^{\lambda(k)}\right|,\left|J^{\lambda(k)}\right|$, respectively. Go to (4).

(4) Calculate

$$
\delta\left(\lambda^{(k)}\right):=\sum_{j \in J_{a}^{\lambda(k)}} d_{j} a_{j}+\sum_{j \in J_{b}^{\lambda(k)}} d_{j} b_{j}+\sum_{j \in J^{\lambda(k)}} d_{j} \hat{x}_{j}-\lambda^{(k)} \sum_{j \in J^{\lambda(k)}} d_{j}^{2}-\alpha^{(k)} .
$$

Go to (5).

(5) If $\delta\left(\lambda^{(k)}\right)=0$ or $J^{\lambda(k)}=\varnothing$, then $\lambda:=\lambda^{(k)}, J_{a}^{\lambda}:=J_{a}^{\lambda} \cup J_{a}^{\lambda(k)}, J_{b}^{\lambda}:=J_{b}^{\lambda} \cup J_{b}^{\lambda(k)}, J^{\lambda}:=J^{\lambda(k)}$, go to $(8)$

else if $\delta\left(\lambda^{(k)}\right)>0$, go to (6)

else if $\delta\left(\lambda^{(k)}\right)<0$, go to (7). 
(6) $x_{j}^{*}:=a_{j}$ for $j \in J_{a}^{\lambda(k)}, \alpha^{(k+1)}:=\alpha^{(k)}-\sum_{j \in J_{a}^{\lambda(k)}} d_{j} a_{j}, J^{(k+1)}:=J^{(k)} \backslash J_{a}^{\lambda(k)}, n^{(k+1)}:=n^{(k)}-$ $\left|J_{a}^{\lambda(k)}\right|, J_{a}^{\lambda}:=J_{a}^{\lambda} \cup J_{a}^{\lambda(k)}, k:=k+1$. Go to (2).

(7) $x_{j}^{*}:=b_{j}$ for $j \in J_{b}^{\lambda(k)}, \alpha^{(k+1)}:=\alpha^{(k)}-\sum_{j \in J_{b}^{\lambda(k)}} d_{j} b_{j}, J^{(k+1)}:=J^{(k)} \backslash J_{b}^{\lambda(k)}, n^{(k+1)}:=n^{(k)}-$ $\left|J_{b}^{\lambda(k)}\right|, J_{b}^{\lambda}:=J_{b}^{\lambda} \cup J_{b}^{\lambda(k)}, k:=k+1$. Go to (2).

(8) $x_{j}^{*}:=a_{j}$ for $j \in J_{a}^{\lambda} ; x_{j}^{*}:=b_{j}$ for $j \in J_{b}^{\lambda} ; x_{j}^{*}:=\hat{x}_{j}-\lambda d_{j}$ for $j \in J^{\lambda}$. Go to (10).

(9) The problem has no optimal solution because $X=\varnothing$ or there does not exist a $\lambda^{*}>0$ satisfying Theorem 2.1 .

(10) End.

3.3. Convergence and complexity of Algorithm 1. The following theorem states convergence of Algorithm 1.

Theorem 3.1. Let $\lambda^{(k)}$ be the sequence generated by Algorithm 1. Then

(i) if $\delta\left(\lambda^{(k)}\right)>0$, then $\lambda^{(k)} \leq \lambda^{(k+1)}$;

(ii) if $\delta\left(\lambda^{(k)}\right)<0$, then $\lambda^{(k)} \geq \lambda^{(k+1)}$.

Proof. Denote by $x_{j}^{(k)}$ the components of $\mathbf{x}^{(k)}=\left(x_{j}\right)_{j \in J^{(k)}}$ at iteration $k$ of implementation of Algorithm 1.

Taking into consideration (3.4), Case 1, Case 2, Case 3, and Step (1) (the sign of $\delta(0)$ ) and Step (2) of Algorithm 1, it follows that $\lambda^{(k)} \geq 0$ for each $k$. Since $x_{j}^{(k)}$ are determined from (2.12), $x_{j}^{(k)}=\hat{x}_{j}-\lambda^{(k)} d_{j}, j \in J^{\lambda(k)}$, substituted in $\sum_{j \in J^{\lambda(k)}} d_{j} x_{j}^{(k)}=\alpha^{(k)}$ at Step (2) of Algorithm 1, and since $\lambda^{(k)} \geq 0, d_{j}>0$, then $\hat{x}_{j} \geq x_{j}^{(k)}$.

(i) Let $\delta\left(\lambda^{(k)}\right)>0$. Using Step (6) of Algorithm 1 (which is performed when $\delta\left(\lambda^{(k)}\right)>0$ ), we get

$$
\sum_{j \in J^{\lambda(k+1)}} d_{j} x_{j}^{(k)} \equiv \sum_{j \in J^{(k+1)}} d_{j} x_{j}^{(k)}=\sum_{j \in J^{(k)} \backslash \bigcup_{a}^{\lambda(k)}} d_{j} x_{j}^{(k)}=\alpha^{(k)}-\sum_{j \in J_{a}^{\lambda(k)}} d_{j} x_{j}^{(k)} .
$$

Let $j \in J_{a}^{\lambda(k)}$. According to definition (2.10) of $J_{a}^{\lambda(k)}$ we have

$$
\frac{\hat{x}_{j}-a_{j}}{d_{j}} \leq \lambda^{(k)}=\frac{\hat{x}_{j}-x_{j}^{(k)}}{d_{j}} .
$$

Multiplying this inequality by $-d_{j}<0$, we obtain $a_{j}-\hat{x}_{j} \geq x_{j}^{(k)}-\hat{x}_{j}$. Therefore $a_{j} \geq$ $x_{j}^{(k)}, j \in J_{a}^{\lambda(k)}$.

Using that $d_{j}>0$ and Step (6), from (3.9) we get

$$
\begin{aligned}
\sum_{j \in J^{\lambda(k+1)}} d_{j} x_{j}^{(k)} & =\alpha^{(k)}-\sum_{j \in J_{a}^{\lambda(k)}} d_{j} x_{j}^{(k)} \geq \alpha^{(k)}-\sum_{j \in J_{a}^{\lambda(k)}} d_{j} a_{j} \\
& =\alpha^{(k+1)}=\sum_{j \in J^{\lambda(k+1)}} d_{j} x_{j}^{(k+1)}
\end{aligned}
$$


Since $d_{j}>0, j=1, \ldots, n$, there exists at least one $j_{0} \in J^{\lambda(k+1)}$ such that $x_{j_{0}}^{(k)} \geq x_{j_{0}}^{(k+1)}$. Then

$$
\lambda^{(k)}=\frac{\hat{x}_{j_{0}}-x_{j_{0}}^{(k)}}{d_{j_{0}}} \leq \frac{\hat{x}_{j_{0}}-x_{j_{0}}^{(k+1)}}{d_{j_{0}}}=\lambda^{(k+1)} .
$$

We have used that the relationship between $\lambda^{(k)}$ and $x_{j}^{(k)}$ is given by (2.12) for $j \in J^{\lambda(k)}$ according to Step (2) of Algorithm 1 and that $\hat{x}_{j} \geq x_{j}^{(k)}, j \in J^{\lambda(k)}$, according to (2.12) with $\lambda^{(k)} \geq 0$ and $d_{j}>0$.

The proof of part (ii) is omitted because it is similar to that of part (i).

Consider the feasibility of $\mathbf{x}^{*}=\left(x_{j}^{*}\right)_{j \in J}$, generated by Algorithm 1 .

Components $x_{j}^{*}=a_{j}, j \in J_{a}^{\lambda}$, and $x_{j}^{*}=b_{j}, j \in J_{b}^{\lambda}$, obviously satisfy (1.4). It follows from

$$
\frac{\hat{x}_{j}-b_{j}}{d_{j}}<\lambda \equiv \frac{\hat{x}_{j}-x_{j}^{*}}{d_{j}}<\frac{\hat{x}_{j}-a_{j}}{d_{j}}, \quad j \in J^{\lambda},
$$

and $d_{j}>0$ that $a_{j}-\hat{x}_{j}<x_{j}^{*}-\hat{x}_{j}<b_{j}-\hat{x}_{j}, j \in J^{\lambda}$. Therefore $a_{j}<x_{j}^{*}<b_{j}$ for $j \in J^{\lambda}$. Hence all $x_{j}^{*}, j=1, \ldots, n$, satisfy $(1.4)$.

We have proved that if $\delta(\lambda)_{\mid \lambda=0} \geq 0$ and $X \neq \varnothing$, where $X$ is defined by (1.3)-(1.4), then there exists a $\lambda^{*} \geq 0$ such that $\delta\left(\lambda^{*}\right)=0$. Since at Step (2) we determine $\lambda^{(k)}$ from the equality $\sum_{j \in J^{\lambda(k)}} d_{j} x_{j}^{(k)}=\alpha^{(k)}$ for each $k$, then (1.3) is satisfied with an equality in this case. Otherwise, if $\delta(0)<0$, then we set $\lambda=0$ (Step (1)) and we have $\sum_{j \in J} d_{j} x_{j}(0)-\alpha \equiv$ $\delta(0)<0$, that is, $(1.3)$ is satisfied as a strict inequality in this case.

Therefore Algorithm 1 generates $\mathbf{x}^{*}$ which is feasible for problem $\left(P^{\leq}\right)$.

Remark 3.2. Theorem 3.1, definitions of $J_{a}^{\lambda}$ (2.10), $J_{b}^{\lambda}$ (2.11), and $J^{\lambda}$ (2.12), and Steps (6), (7), (8) of Algorithm 1 allow us to assert that $J_{a}^{\lambda(k)} \subseteq J_{a}^{\lambda(k+1)}, J_{b}^{\lambda(k)} \subseteq J_{b}^{\lambda(k+1)}$, and $J^{\lambda(k)} \supseteq$ $J^{\lambda(k+1)}$. This means that if $j$ belongs to current set $J_{a}^{\lambda(k)}$, then $j$ belongs to the next index set $J_{a}^{\lambda(k+1)}$ and, therefore, to the optimal index set $J_{a}^{\lambda}$, the same holds true about the sets $J_{b}^{\lambda(k)}$ and $J_{b}^{\lambda}$. Therefore $\lambda^{(k)}$ "converges" to the optimal $\lambda$ of Theorem 2.1 and $J_{a}^{\lambda(k)}, J_{b}^{\lambda(k)}$, $J^{\lambda(k)}$ "converge" to the optimal index sets $J_{a}^{\lambda}, J_{b}^{\lambda}, J^{\lambda}$, respectively. This means that calculation of $\lambda$, operations $x_{j}^{*}:=a_{j}, j \in J_{a}^{\lambda(k)}$ (Step (6)), $x_{j}^{*}:=b_{j}, j \in J_{b}^{\lambda(k)}$ (Step (7)), and the construction of $J_{a}^{\lambda}, J_{b}^{\lambda}, J^{\lambda}$ are in accordance with Theorem 2.1.

At each iteration, Algorithm 1 determines the value of at least one variable (Steps (6), $(7),(8))$, and at each iteration, we solve a problem of the form $\left(P^{\leq}\right)$but of less dimension (Steps (2)-(7)). Therefore Algorithm 1 is finite and it converges with at most $n=|J|$ iterations, that is, the iteration complexity of Algorithm 1 is $O(n)$.

Step (0) takes time $O(n)$. Step (1) (construction of sets $J_{a}^{0}, J_{b}^{0}, J^{0}$, calculation of $\delta(0)$, and checking whether $X$ is empty) also takes time $\mathcal{O}(n)$. The calculation of $\lambda^{(k)}$ requires constant time (Step (2)). Step (3) takes $O(n)$ time because of the construction of $J_{a}^{\lambda(k)}$, $J_{b}^{\lambda(k)}, J^{\lambda(k)}$. Step (4) also requires $O(n)$ time and Step (5) requires constant time. Each of Steps (6), (7), and (8) takes time which is bounded by $O(n)$ : at these steps we assign the final value to some of $x_{j}$, and since the number of all $x_{j}$ 's is $n$, then Steps (6), (7), and (8) 
take time $\mathrm{O}(n)$. Hence the algorithm has $\mathrm{O}\left(n^{2}\right)$ running time and it belongs to the class of strongly polynomially bounded algorithms.

As the computational experiments show, the number of iterations of the algorithm performance is not only at most $n$ but it is much, much less than $n$ for large $n$. In fact, this number does not depend on $n$ but only on the three index sets defined by (2.10), (2.11), (2.12). In practice, Algorithm 1 has $\mathcal{O}(n)$ running time.

3.4. Algorithm 2 (for problem $\left(P^{=}\right)$) and its convergence. After analysis of the optimal solution to problem $\left(P^{=}\right)$, similar to that to problem $\left(P^{\leq}\right)$, we suggest the following algorithm for solving problem $\left(P^{=}\right)$.

Algorithm 2 (for problem $\left(P^{=}\right)$).

(1) (Initialization). $J:=\{1, \ldots, n\}, k:=0, \alpha^{(0)}:=\alpha, n^{(0)}:=n, J^{(0)}:=J, J_{a}^{\lambda}:=\varnothing, J_{b}^{\lambda}:=\varnothing$, initialize $\hat{x}_{j}, j \in J$. If $\sum_{j \in J} d_{j} a_{j} \leq \alpha \leq \sum_{j \in J} d_{j} b_{j}$, go to (2), else go to (9).

(2) $J^{\lambda(k)}:=J^{(k)}$. Calculate $\lambda^{(k)}$ by using the explicit expression of $\lambda$. Go to (3).

(3) Construct the sets $J_{a}^{\lambda(k)}, J_{b}^{\lambda(k)}, J^{\lambda(k)}$ through (2.30), (2.31), (2.32) (with $j \in J^{(k)}$ instead of $j \in J)$ and find their cardinalities $\left|J_{a}^{\lambda(k)}\right|,\left|J_{b}^{\lambda(k)}\right|,\left|J^{\lambda(k)}\right|$. Go to (4).

Steps (4)-(8) are the same as Steps (4)-(8) of Algorithm 1, respectively.

(9) Problem $\left(P^{=}\right)$has no optimal solution because the feasible set $X(1.5)-(1.6)$ is empty.

(10) End.

A theorem analogous to Theorem 3.1 holds for Algorithm 2 which guarantees the “convergence" of $\lambda^{(k)}, J^{\lambda(k)}, J_{a}^{\lambda(k)}, J_{b}^{\lambda(k)}$ to the optimal $\lambda, J^{\lambda}, J_{a}^{\lambda}, J_{b}^{\lambda}$, respectively.

Theorem 3.3. Let $\lambda^{(k)}$ be the sequence generated by Algorithm 2. Then

(i) if $\delta\left(\lambda^{(k)}\right)>0$, then $\lambda^{(k)} \leq \lambda^{(k+1)}$;

(ii) if $\delta\left(\lambda^{(k)}\right)<0$, then $\lambda^{(k)} \geq \lambda^{(k+1)}$.

The proof of Theorem 3.3 is omitted because it is similar to that of Theorem 3.1.

It can be proved that Algorithm 2 has $\mathcal{O}\left(n^{2}\right)$ running time, and point $\mathbf{x}^{*}=\left(x_{1}^{*}, \ldots, x_{n}^{*}\right)$ generated by this algorithm is feasible for problem $\left(P^{=}\right)$, which is an assumption of Theorem 2.2.

The following algorithm for solving problem $\left(P^{\geq}\right)$is based on Theorem 2.3 and Algorithm 2.

\subsection{Algorithm 3 (for problem $\left(P^{\geq}\right)$)}

Algorithm 3.

(1) (Initialization). $J:=\{1, \ldots, n\}, k:=0, J^{(0)}:=J, \alpha^{(0)}:=\alpha, n^{(0)}:=n, J_{a}^{\lambda}:=\varnothing J_{b}^{\lambda}:=\varnothing$, initialize $\hat{x}_{j}, j \in J$. If $\sum_{j \in J} d_{j} a_{j} \leq \alpha \leq \sum_{j \in J} d_{j} b_{j}$, then go to (2), else go to (9).

Steps (2)-(7) are the same as Steps (2)-(7) of Algorithm 2, respectively.

(8) If $\lambda \leq 0$, then $x_{j}^{\geq}:=a_{j}$ for $j \in J_{a}^{\lambda}, x_{j}^{\geq}:=b_{j}$ for $j \in J_{b}^{\lambda}, x_{j}^{\geq}:=\hat{x}_{j}-\lambda d_{j}$ for $j \in J^{\lambda}$, go to $(10)$ else if $\lambda>0$, then

$$
x_{j}^{\geq}:=b_{j} \text { for } j \in J_{b}^{\lambda} \text {, }
$$


424 Polynomial algorithms for projecting a point in $\mathbb{R}^{n}$

$$
\begin{aligned}
& x_{j}^{\geq}:=\min \left\{b_{j}, \hat{x}_{j}\right\} \text { for } j \in J^{\lambda}, \\
& \text { if } j \in J_{a}^{\lambda} \text { and } a_{j}<\hat{x}_{j}, \text { then } x_{j}^{\geq}:=\min \left\{b_{j}, \hat{x}_{j}\right\} \\
& \text { else if } j \in J_{a}^{\lambda} \text { and } a_{j} \geq \hat{x}_{j}, \text { then } x_{j}^{\geq}:=a_{j} ; \\
& \text { go to }(10) .
\end{aligned}
$$

(9) Problem $\left(P^{\geq}\right)$has no optimal solution because $X=\varnothing$, where $X$ is defined by (1.7)(1.8), or there do not exist $x_{j}^{*} \in\left[a_{j}, b_{j}\right], j \in J$, such that $\sum_{j \in J} d_{j} x_{j}^{*}=\alpha$.

(10) End.

Since Algorithm 3 is based on Theorem 2.3 and Algorithm 2 and since the "iterative" steps (2)-(7) of Algorithm 2 and Algorithm 3 are the same, then "convergence" of Algorithm 3 follows from Theorem 3.3 as well. Because of the same reason, computational complexity of Algorithm 3 is the same as that of Algorithm 2.

3.6. Commentary. Methods proposed for solving problems $\left(P^{\leq}\right),\left(P^{=}\right)$, and $\left(P^{\geq}\right)$are first-order methods because they use values of the first derivatives (see (2.3)) of the objective function $c(\mathbf{x}) \equiv \sum_{j=1}^{n} c_{j}\left(x_{j}\right) \equiv(1 / 2) \sum_{j=1}^{n}\left(x_{j}-\hat{x}_{j}\right)^{2}$. Also, they are dual variables saddle point methods because they are based on "convergence" with respect to the Lagrange multiplier (dual variable) $\lambda$ associated with the single constraint (1.3) (resp., constraint (1.5) or (1.7)). Moreover, they are exact methods because there are only round-off errors and there is no error of the methods.

Methods suggested in this paper, due to specificity of problems solved, are less restrictive than other methods for solving general convex quadratic programming problems, such as active set methods and gradient projection methods, with respect to dimension (number of variables) of the problem, convergence conditions, subproblems to be solved at each iteration, and so forth.

\section{Extensions}

4.1. Theoretical aspects. If it is allowed that $d_{j}=0$ for some $j$ in problems $\left(P^{\leq}\right),\left(P^{=}\right)$, and $\left(P^{\geq}\right)$, then, for such indices $j$, we cannot construct the expressions $\left(\hat{x}_{j}-a_{j}\right) / d_{j}$ and $\left(\hat{x}_{j}-b_{j}\right) / d_{j}$ by means of which we define sets $J_{a}^{\lambda}, J_{b}^{\lambda}, J^{\lambda}$ for the corresponding problem. In such cases, $x_{j}$ 's are not involved in (1.3) (resp., in (1.5) or in (1.7)) for such indices $j$. It turns out that we can cope with this difficulty and solve problems $\left(P^{\leq}\right),\left(P^{=}\right),\left(P^{\geq}\right)$with $d_{j}=0$ for some $j$ 's.

Denote

$$
J=\{1, \ldots, n\}, \quad Z 0=\left\{j \in J: d_{j}=0\right\}
$$

Here " 0 " means the "computer zero." In particular, when $J=Z 0$ and $\alpha=0, X$ is defined only by (1.4) (resp., by (1.6), by (1.8)).

Theorem 4.1 (characterization of the optimal solution to problem $\left(P^{\leq}\right)$: an extended version). Problem $\left(P^{\leq}\right)$can be decomposed into two subproblems: $\left(P 1^{\leq}\right)$for $j \in Z 0$ and $\left(P 2^{\leq}\right)$for $j \in J \backslash Z 0$. 
The optimal solution to $\left(P 1^{\leq}\right)$is

$$
x_{j}^{*}= \begin{cases}a_{j}, & j \in Z 0, \hat{x}_{j} \leq a_{j}, \\ b_{j}, & j \in Z 0, \hat{x}_{j} \geq b_{j}, \\ \hat{x}_{j}, & j \in Z 0, a_{j}<\hat{x}_{j}<b_{j},\end{cases}
$$

that is, the subproblem $\left(P 1^{\leq}\right)$itself is decomposed into $n_{0} \equiv|Z 0|$ independent problems. The optimal solution to $\left(P 2^{\leq}\right)$is given by (2.10), (2.11), (2.12) with $J:=J \backslash Z 0$.

Proof

Necessity. Let $\mathbf{x}^{*}=\left(x_{j}^{*}\right)_{j \in J}$ be the optimal solution to $\left(P^{\leq}\right)$.

(1) Let $j \in Z 0$, that is, $d_{j}=0$. The KKT conditions are

$$
x_{j}^{*}-\hat{x}_{j}-u_{j}+v_{j}=0, \quad j \in Z 0 \quad \text { from (2.3) and (2.4)-(2.9). }
$$

(a) If $x_{j}^{*}=a_{j}$, then $u_{j} \geq 0, v_{j}=0$. It follows from (4.3) that $x_{j}^{*}-\hat{x}_{j}=u_{j} \geq 0$; that is, $x_{j}^{*} \equiv a_{j} \geq \hat{x}_{j}$.

(b) If $x_{j}^{*}=b_{j}$, then $u_{j}=0, v_{j} \geq 0$. Therefore $x_{j}^{*}-\hat{x}_{j}=-v_{j} \leq 0$; that is, $x_{j}^{*} \equiv b_{j} \leq$ $\widehat{x}_{j}$.

(c) If $a_{j}<x_{j}^{*}<b_{j}$, then $u_{j}=v_{j}=0$. Therefore $x_{j}^{*}-\hat{x}_{j}=0$; that is, $x_{j}^{*}=\hat{x}_{j}$.

(2) Components of the optimal solution to $\left(P 2^{\leq}\right)$are obtained by using the same approach as that of the proof of Theorem 2.1(i) but with the reduced index set $J:=J \backslash Z 0$.

Sufficiency. Conversely, let $\mathbf{x}^{*} \in X$ and let the components of $\mathbf{x}^{*}$ satisfy (4.2) for $j \in Z 0$, and (2.10), (2.11), (2.12) with $J:=J \backslash Z 0$. Set

$$
\begin{aligned}
& u_{j}=v_{j}=0 \quad \text { for } a_{j}<x_{j}^{*}<b_{j}, j \in Z 0, \\
& u_{j}=a_{j}-\hat{x}_{j}, \quad v_{j}=0 \quad \text { for } x_{j}^{*}=a_{j}, j \in Z 0, \\
& u_{j}=0, \quad v_{j}=\hat{x}_{j}-b_{j} \quad \text { for } x_{j}^{*}=b_{j}, j \in Z 0 .
\end{aligned}
$$

If $\lambda>0$, set

$$
\begin{aligned}
& \lambda=\frac{\hat{x}_{j}-x_{j}^{*}}{d_{j}}=\lambda\left(\mathbf{x}^{*}\right)(>0) \quad \text { from }(2.12), \\
& u_{j}=v_{j}=0 \quad \text { for } a_{j}<x_{j}^{*}<b_{j}, j \in J \backslash Z 0, \\
& u_{j}=a_{j}-\hat{x}_{j}+\lambda d_{j}(\geq 0), \quad v_{j}=0 \quad \text { for } x_{j}^{*}=a_{j}, j \in J \backslash Z 0, \\
& u_{j}=0, \quad v_{j}=\hat{x}_{j}-b_{j}-\lambda d_{j}(\geq 0) \quad \text { for } x_{j}^{*}=b_{j}, j \in J \backslash Z 0 .
\end{aligned}
$$

If $\lambda=0$, set

$$
\begin{array}{ll}
u_{j}=v_{j}=0 \quad \text { for } a_{j}<x_{j}^{*}<b_{j}, j \in J \backslash Z 0, \\
u_{j}=a_{j}-\hat{x}_{j}(\geq 0), \quad v_{j}=0 & \text { for } x_{j}^{*}=a_{j}, j \in J \backslash Z 0, \\
u_{j}=0, \quad v_{j}=\hat{x}_{j}-b_{j}(\geq 0) & \text { for } x_{j}^{*}=b_{j}, j \in J \backslash Z 0 .
\end{array}
$$


It can be verified that $\mathbf{x}^{*}, \lambda, u_{j}, v_{j}, j \in J$, satisfy the KKT conditions (4.3), (2.4)-(2.9). Then $\mathbf{x}^{*}$ with components (4.2), for $j \in Z 0$, and (2.10), (2.11), (2.12), with $J:=J \backslash Z 0$, is the optimal solution to problem $\left(P^{\leq}\right)=\left(P 1^{\leq}\right) \cup\left(P 2^{\leq}\right)$.

An analogous result holds for problem $\left(P^{=}\right)$.

Theorem 4.2 (characterization of the optimal solution to problem $\left(P^{=}\right)$: an extended version). Problem $\left(P^{=}\right)$can be decomposed into two subproblems: $\left(P 1^{=}\right)$for $j \in Z 0$ and $\left(P 2^{=}\right)$for $j \in J \backslash Z 0$.

The optimal solution to $\left(P 1^{=}\right)$is also given by (4.2). The optimal solution to $\left(P 2^{=}\right)$is given by (2.30), (2.31), (2.32) with $J:=J \backslash Z 0$.

The proof of Theorem 4.2 is omitted because it repeats in part the proofs of Theorems 2.1 and 4.1 .

Thus, with the use of Theorems 4.1 and 4.2, we can express components of the optimal solutions to problems $\left(P^{\leq}\right),\left(P^{=}\right)$, and $\left(P^{\geq}\right)$without the necessity of constructing the expressions $\left(\hat{x}_{j}-a_{j}\right) / d_{j}$ and $\left(\hat{x}_{j}-b_{j}\right) / d_{j}$ with $d_{j}=0$.

Since Theorem 2.3 and Algorithm 3 are based on the sets of indices $J_{a}^{\lambda}, J_{b}^{\lambda}, J^{\lambda}$ of problem $\left(P^{=}\right)$, Theorem 4.2 solves the problem of decomposition of problem $\left(P^{\geq}\right)$as well.

4.2. Computational aspects. Algorithms 1, 2, and 3 are also applicable in cases when $a_{j}=-\infty$ for some $j, 1 \leq j \leq n$ and/or $b_{j}=\infty$ for some $j, 1 \leq j \leq n$. However, if we use the computer values of $-\infty$ and $+\infty$ at the first step of the algorithms to check whether the corresponding feasible region is empty or nonempty and at Step (3) in the expressions $\left(\hat{x}_{j}-x_{j}\right) / d_{j}$ with $x_{j}=-\infty$ and/or $x_{j}=+\infty$, by means of which we construct sets $J_{a}^{\lambda}, J_{b}^{\lambda}, J^{\lambda}$, this could sometimes lead to arithmetic overflow. If we use other values of $-\infty$ and $+\infty$ with smaller absolute values than those of the computer values of $-\infty$ and $+\infty$, it would lead to inconvenience and dependence on the data of the particular problems. To avoid these difficulties and to take into account the problems considered above, it is convenient to do the following.

Construct the sets of indices

$$
\begin{aligned}
S V N & =\left\{j \in J \backslash Z 0: a_{j}>-\infty, b_{j}<+\infty\right\}, \\
S V 1 & =\left\{j \in J \backslash Z 0: a_{j}>-\infty, b_{j}=+\infty\right\}, \\
S V 2 & =\left\{j \in J \backslash Z 0: a_{j}=-\infty, b_{j}<+\infty\right\}, \\
S V & =\left\{j \in J \backslash Z 0: a_{j}=-\infty, b_{j}=+\infty\right\} .
\end{aligned}
$$

It is obvious that $Z 0 \cup S V \cup S V 1 \cup S V 2 \cup S V N=J$, that is, the set $J \backslash Z 0$ is partitioned into the four subsets $S V N, S V 1, S V 2, S V$ defined above.

When programming the algorithms, we use computer values of $-\infty$ and $+\infty$ for constructing the sets $S V N, S V 1, S V 2, S V$.

In order to construct the sets $J_{a}^{\lambda}, J_{b}^{\lambda}$, $J^{\lambda}$ without the necessity of calculating the values $\left(\hat{x}_{j}-x_{j}\right) / d_{j}$ with $x_{j}=-\infty$ or $+\infty$, except for the sets $J, Z 0, S V, S V 1, S V 2, S V N$, we need some subsidiary sets defined as follows. 
For $S V N$,

$$
\begin{aligned}
& J^{\lambda S V N}=\left\{j \in S V N: \frac{\hat{x}_{j}-b_{j}}{d_{j}}<\lambda<\frac{\hat{x}_{j}-a_{j}}{d_{j}}\right\}, \\
& J_{a}^{\lambda S V N}=\left\{j \in S V N: \lambda \geq \frac{\hat{x}_{j}-a_{j}}{d_{j}}\right\}, \\
& J_{b}^{\lambda S V N}=\left\{j \in S V N: \lambda \leq \frac{\hat{x}_{j}-b_{j}}{d_{j}}\right\} ;
\end{aligned}
$$

for $S V 1$,

$$
\begin{aligned}
& J^{\lambda S V 1}=\left\{j \in S V 1: \lambda<\frac{\hat{x}_{j}-a_{j}}{d_{j}}\right\}, \\
& J_{a}^{\lambda S V 1}=\left\{j \in S V 1: \lambda \geq \frac{\hat{x}_{j}-a_{j}}{d_{j}}\right\} ;
\end{aligned}
$$

for $S V 2$,

$$
\begin{aligned}
& J^{\lambda S V 2}=\left\{j \in S V 2: \lambda>\frac{\hat{x}_{j}-b_{j}}{d_{j}}\right\}, \\
& J_{b}^{\lambda S V 2}=\left\{j \in S V 2: \lambda \leq \frac{\hat{x}_{j}-b_{j}}{d_{j}}\right\} ;
\end{aligned}
$$

for $S V$,

$$
J^{\lambda S V}=S V
$$

Then

$$
\begin{aligned}
J^{\lambda} & :=J^{\lambda S V N} \cup J^{\lambda S V 1} \cup J^{\lambda S V 2} \cup J^{\lambda S V}, \\
J_{a}^{\lambda} & :=J_{a}^{\lambda S V N} \cup J_{a}^{\lambda S V 1}, \\
J_{b}^{\lambda} & :=J_{b}^{\lambda S V N} \cup J_{b}^{\lambda S V 2} .
\end{aligned}
$$

We use the sets $J^{\lambda}, J_{a}^{\lambda}, J_{b}^{\lambda}$ (4.12) as the corresponding sets with the same names in Algorithms 1, 2, and 3.

With the use of results of this section, Steps (0), (1), and (3) of Algorithm 1 can be modified, respectively as follows.

\section{About Algorithm 1.}

Step $(0)^{1}$. (Initialization). $J:=\{1, \ldots, n\}, k:=0, \alpha^{(0)}:=\alpha, n^{(0)}:=n$, $J^{(0)}:=J, J_{a}^{\lambda}:=\varnothing, J_{b}^{\lambda}:=\varnothing$, initialize $\hat{x}_{j}, j \in J$.

Construct the set $Z 0$. If $j \in Z 0$, then

if $\hat{x}_{j} \leq a_{j}$, then $x_{j}^{*}:=a_{j}$,

else if $\hat{x}_{j} \geq b_{j}$, then $x_{j}^{*}:=b_{j}$, else if $a_{j}<\hat{x}_{j}<b_{j}$, then $x_{j}^{*}:=\hat{x}_{j}$. 
If $J=Z 0$ and $\alpha=0$, go to Step (10)

else if $J=Z 0$ and $\alpha \neq 0$, go to Step (9).

Set $J:=J \backslash Z 0, J^{(0)}:=J, n^{(0)}:=n-|Z 0|$.

Construct the sets $S V N, S V 1, S V 2, S V$.

If $S V N \cup S V 1=J$, then

if $\sum_{j \in J} d_{j} a_{j} \leq \alpha$, go to Step (1)

else go to Step (9) (feasible region $X$ is empty)

else if $S V 2 \cup S V \neq \varnothing$, then

if $S V N \cup S V 1 \neq \varnothing$, then

if $\sum_{j \in S V N \cup S V 1} d_{j} a_{j} \leq \alpha$, go to Step (1)

else go to Step (9) (feasible region is empty)

else go to Step (1) (feasible region is always nonempty).

Step $(1)^{1}$. Construct the sets $J^{0 S V N}, J_{a}^{0 S V N}, J_{b}^{0 S V N}, J^{0 S V 1}, J_{a}^{0 S V 1}, J^{0 S V 2}, J_{b}^{0 S V 2}, J^{0 S V}$ (for $\lambda=0$ ). Construct the sets $J_{a}^{0}, J_{b}^{0}, J^{0}$ through (4.12). Calculate

$$
\delta(0):=\sum_{j \in J_{a}^{0}} d_{j} a_{j}+\sum_{j \in J_{b}^{0}} d_{j} b_{j}+\sum_{j \in J^{0}} d_{j} \hat{x}_{j}-\alpha .
$$

If $\delta(0) \leq 0$, then $\lambda:=0$, go to Step (8)

else if $\delta(0)>0$, then

if $S V 2 \cup S V N=J$, then

if $\alpha \leq \sum_{j \in J} d_{j} b_{j}$, go to Step (2)

else go to Step (9) (there does not exist a $\lambda^{*}>0$ such that $\delta\left(\lambda^{*}\right)=0$ )

else if $S V 1 \cup S V \neq \varnothing$, go to Step (2) (there exists a $\lambda^{*}>0$ such that $\delta\left(\lambda^{*}\right)=0$ ).

Step (3) ${ }^{1}$. Construct the sets $J^{\lambda S V N}, J_{a}^{\lambda S V N}, J_{b}^{\lambda S V N}, J^{\lambda S V 1}, J_{a}^{\lambda S V 1}, J^{\lambda S V 2}, J_{b}^{\lambda S V 2}, J^{\lambda S V}$ (with $J^{(k)}$ instead of $J$ ).

Construct the sets $J_{a}^{\lambda(k)}, J_{b}^{\lambda(k)}, J^{\lambda(k)}$ by using (4.12) and find their cardinalities $\left|J_{a}^{\lambda(k)}\right|$, $\left|J_{b}^{\lambda(k)}\right|,\left|J^{\lambda(k)}\right|$, respectively. Go to Step (4).

Similarly, we can modify Steps (1) and (3) of both Algorithm 2 and Algorithm 3.

Modifications of the algorithms connected with theoretical and computational aspects do not influence their computational complexity, which has been discussed in Section 3, because these modifications do not affect the "iterative" steps of algorithms.

\section{Computational experiments}

In this section, we present results (Table 5.1) of some numerical experiments obtained by applying algorithms suggested in this paper to problems under consideration. The computations were performed on an Intel Pentium II Celeron Processor $466 \mathrm{MHz} / 128 \mathrm{MB}$ SDRAM IBM PC compatible. Each problem was run 30 times. Coefficients $d_{j}>0, j=$ $1, \ldots, n$, and data $\hat{\mathbf{x}}=\left(\hat{x}_{1}, \ldots, \hat{x}_{n}\right)$ were randomly generated according to uniform and Gaussian (normal) distribution. It is also possible to use values generated according to 
Table 5.1

\begin{tabular}{l|cccc}
\hline Problem & \multicolumn{5}{|c}{$\left(P^{\leq}\right)$} \\
\hline Number of variables & $n=1200$ & $n=1500$ & $n=5000$ & $n=10000$ \\
Average number of iterations & 2.09 & 2.11 & 3.58 & 5.53 \\
Average run time (in seconds) & 0.0001 & 0.00011 & 0.00026 & 0.00055 \\
\hline Problem & \multicolumn{5}{|c}{$\left(P^{=}\right)$} \\
\hline Number of variables & $n=1200$ & $n=1500$ & $n=5000$ & $n=10000$ \\
Average number of iterations & 2.07 & 2.10 & 3.67 & 5.64 \\
Average run time (in seconds) & 0.00009 & 0.0001 & 0.00032 & 0.00057 \\
\hline Problem & \multicolumn{5}{c}{$\left(P^{\geq}\right)$} \\
\hline Number of variables & $n=1200$ & $n=1500$ & $n=5000$ & $n=10000$ \\
Average number of iterations & 2.09 & 2.12 & 3.78 & 5.57 \\
Average run time (in seconds) & 0.000101 & 0.00011 & 0.00037 & 0.00061 \\
\hline
\end{tabular}

other distributions as well as data of real problems. Efficiency of Algorithms 1, 2, and 3 does not depend on the data.

When $n<1200$, the run time of the algorithms is so small that the timer does not recognize the corresponding value from its computer zero. In such cases the timer displays 0 seconds.

Effectiveness of algorithms for problems $\left(P^{\leq}\right),\left(P^{=}\right)$, and $\left(P^{\geq}\right)$has been tested by many other examples. As we can observe, the (average) number of iterations is much less than the number of variables $n$ for large $n$.

We provide below the solution of two simple particular problems of the form $\left(P^{=}\right)$ obtained by using the approach suggested in this paper.

Example 5.1. Find the projection of $\widehat{\mathbf{x}}=(55,12,15,85,30)$ on a set defined as follows:

$$
\begin{gathered}
x_{1}+x_{2}+2 x_{3}+3 x_{4}+x_{5}=200 \\
0 \leq x_{1} \leq 50 \\
0 \leq x_{2} \leq 7 \\
0 \leq x_{3} \leq 7 \\
0 \leq x_{4} \leq 80 \\
0 \leq x_{5} \leq 25
\end{gathered}
$$

The projection of $\hat{\mathbf{x}}$ on this set is

$$
\mathbf{x}^{*}=(42.27,0.0,0.0,46.81,17.27)
$$

The Euclidean distance between $\widehat{\mathbf{x}}$ and $\mathbf{x}^{*}$ is

$$
\operatorname{dist}\left(\mathbf{x}^{*}, \hat{\mathbf{x}}\right)=\left\|\mathbf{x}^{*}-\hat{\mathbf{x}}\right\|=\left(\sum_{j=1}^{5}\left(x_{j}^{*}-\hat{x}_{j}\right)^{2}\right)^{1 / 2}=46.37691 .
$$


Example 5.2. Find the projection of $\widehat{\mathbf{x}}=(2,3,1,2)$ on a feasible region defined as follows:

$$
\begin{gathered}
x_{1}+x_{2}+x_{3}+x_{4}=1, \\
0 \leq x_{j} \leq 1, \quad j=1,2,3,4 .
\end{gathered}
$$

The projection of $\hat{\mathbf{x}}$ on this region is

$$
\mathbf{x}^{*}=(0,1,0,0)
$$

The Euclidean distance between $\hat{\mathbf{x}}$ and $\mathbf{x}^{*}$ is

$$
\operatorname{dist}\left(\mathbf{x}^{*}, \widehat{\mathbf{x}}\right)=\left\|\mathbf{x}^{*}-\hat{\mathbf{x}}\right\|=\left(\sum_{j=1}^{4}\left(x_{j}^{*}-\hat{x}_{j}\right)^{2}\right)^{1 / 2}=3.60555
$$

\section{Concluding remarks}

The approach proposed in this paper could be modified for strictly convex quadratic objective functions and, more generally, for strictly convex separable objective functions.

\section{Acknowledgment}

The author is grateful to the anonymous referees for their comments and suggestions.

\section{References}

[1] D. P. Bertsekas, Projected Newton methods for optimization problems with simple constraints, SIAM J. Control Optim. 20 (1982), no. 2, 221-246.

[2] G. R. Bitran and A. C. Hax, Disaggregation and resource allocation using convex knapsack problems with bounded variables, Management Sci. 27 (1981), no. 4, 431-441.

[3] P. Brucker, $A n O(n)$ algorithm for quadratic knapsack problems, Oper. Res. Lett. 3 (1984), no. 3, $163-166$.

[4] A. Charnes and W. W. Cooper, The theory of search: optimum distribution of search effort, Management Sci. 5 (1958), 44-50.

[5] S. M. Grzegórski, Orthogonal projections on convex sets for Newton-like methods, SIAM J. Numer. Anal. 22 (1985), no. 6, 1208-1219.

[6] M. Held, P. Wolfe, and H. P. Crowder, Validation of subgradient optimization, Math. Programming 6 (1974), 62-88.

[7] N. Katoh, T. Ibaraki, and H. Mine, A polynomial time algorithm for the resource allocation problem with a convex objective function, J. Oper. Res. Soc. 30 (1979), no. 5, 449-455.

[8] H. Luss and S. K. Gupta, Allocation of effort resources among competing activities, Operations Res. 23 (1975), no. 2, 360-366.

[9] C. Michelot, A finite algorithm for finding the projection of a point onto the canonical simplex of $\mathbf{R}^{n}$, J. Optim. Theory Appl. 50 (1986), no. 1, 195-200.

[10] R. T. Rockafellar and R. J.-B. Wets, A note about projections in the implementation of stochastic quasigradient methods, Numerical Techniques for Stochastic Optimization (Yu. Ermoliev and R. J.-B. Wets, eds.), Springer Ser. Comput. Math., vol. 10, Springer, Berlin, 1988, pp. 385-392.

[11] S. M. Stefanov, On the implementation of stochastic quasigradient methods to some facility location problems, Yugosl. J. Oper. Res. 10 (2000), no. 2, 235-256. 
[12] , Convex separable minimization subject to bounded variables, Comput. Optim. Appl. 18 (2001), no. 1, 27-48.

[13] Separable Programming. Theory and Methods, Applied Optimization, vol. 53, Kluwer Academic Publishers, Dordrecht, 2001.

[14] Convex separable minimization problems with a linear constraint and bounds on the variables, Applications of Mathematics in Engineering and Economics (Sozopol, 2001), Heron Press, Sofia, 2002, pp. 392-402.

[15] P. H. Zipkin, Simple ranking methods for allocation of one resource, Management Sci. 26 (1980), no. $1,34-43$.

Stefan M. Stefanov: Department of Mathematics, South-West University “Neofit Rilski,” 2700 Blagoevgrad, Bulgaria

E-mail address: stefm@aix.swu.bg 


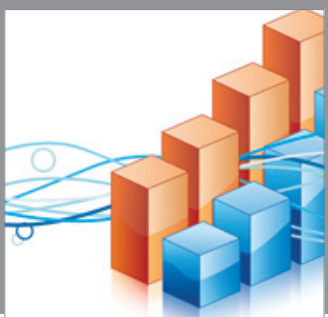

Advances in

Operations Research

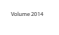

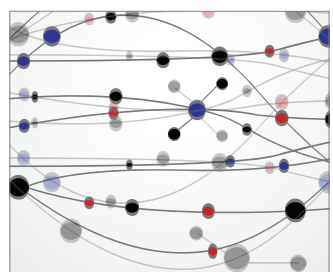

\section{The Scientific} World Journal
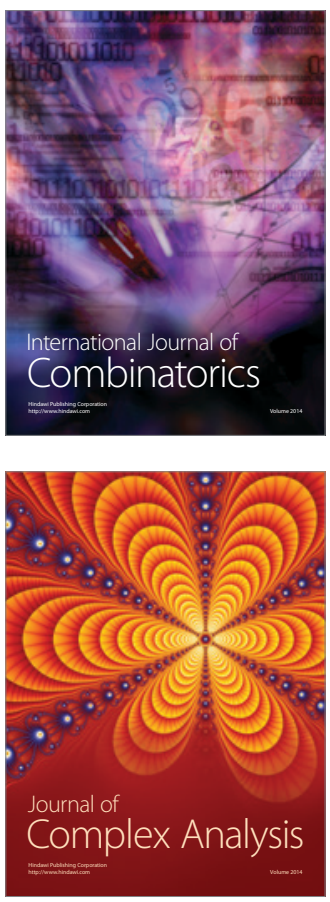

International Journal of

Mathematics and

Mathematical

Sciences
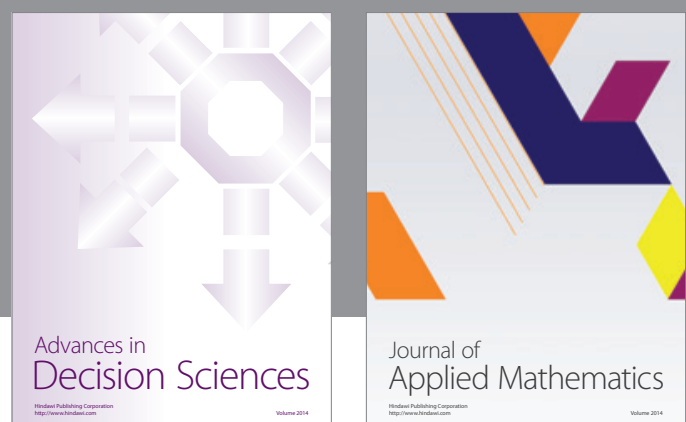

Journal of

Applied Mathematics
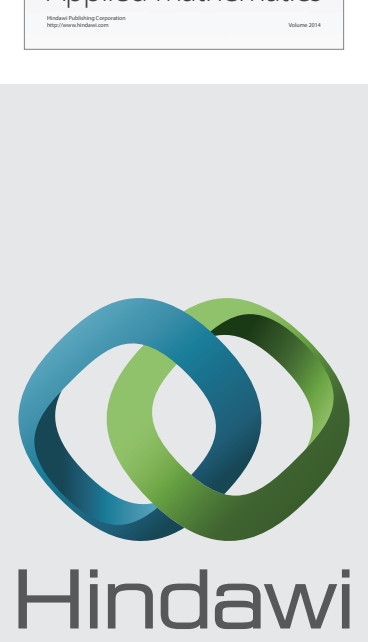

Submit your manuscripts at http://www.hindawi.com
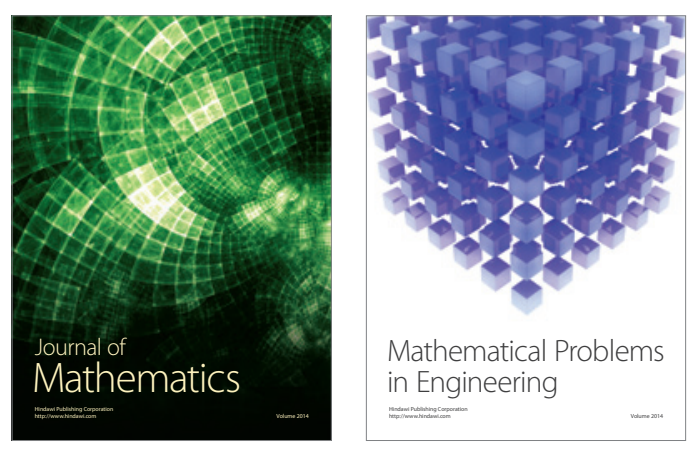

Mathematical Problems in Engineering
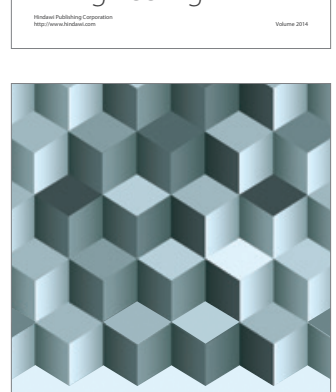

Journal of

Function Spaces
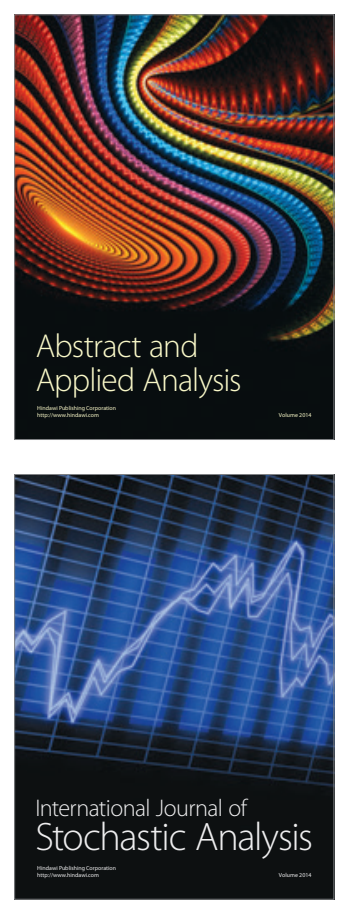

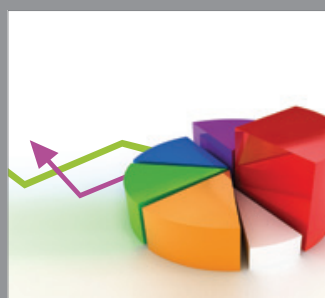

ournal of

Probability and Statistics

Promensencen
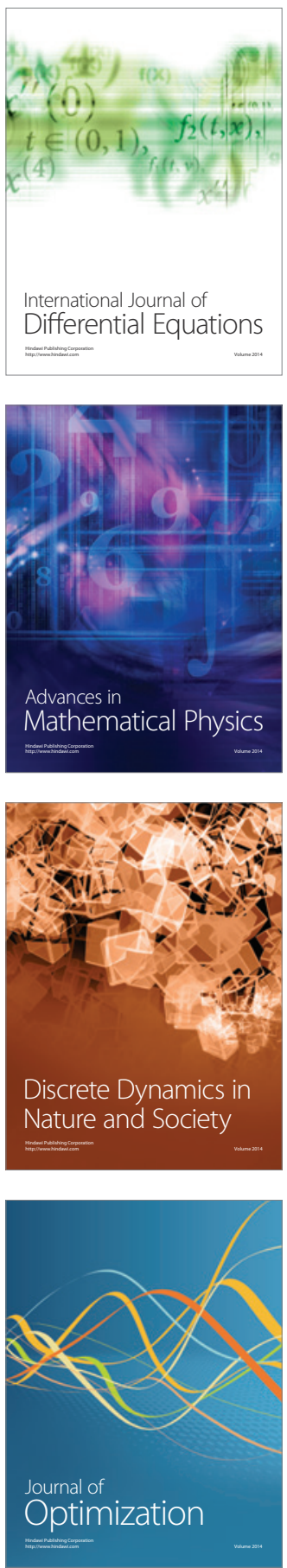